\title{
Estimating COVID-19 Early Pandemic Severity in Indian Context
}

\author{
Manisha Mandal', Shyamapada Mandal ${ }^{2 *}$ \\ ${ }^{1}$ Department of Physiology, MGM Medical College, Kishanganj-855107, India
}

${ }^{2}$ Laboratory of Microbiology and Experimental Medicine, Department of Zoology, University of Gour Banga, Malda-732103, India

${ }^{*}$ Correspondence to: Prof. Shyamapada Mandal, Laboratory of Microbiology and Experimental Medicine, Department of Zoology, University of Gour Banga, Malda-732103, India. E-mail: samtropmed@gmail.com; sam.micro11@ugb.ac.in

\section{ABSTRACT \\ Objective: To explore the early pandemic severity of COVID-19 in India in terms of various case fatality rate (CFR) estimates.}

Methods: Various COVID-19 fatalities: confirmed CFR (CCFR), asymptomatic CFR (aCFR), symptomatic CFR (sCFR), and hospitalized CFR (HFR) were estimated along with relative susceptibility of developing symptoms (RSODS) and relative susceptibility of developing infection (RSODI) determination for Psym (probability of developing symptoms) $0.50,0.75$, and 0.95 each for all age groups.

Results:The CCFR, aCFR, SCFR, and HFR estimates were 2.32\%(2.05-2.59), 0.14\%(0.12-0.16), 0.32\%(0.27-0.36), 1.86\%(1.64-2.07) respectively. The RSODS and RSODI of COVID-19 were $\sim 33$ times higher among people aged <45 years. The RSODS estimates were 1.97 (0.47-3.47), 0.62 (0.15-1.09), 0.29 (0.07-0.52), 0.06 (0.02-0.10) respectively, for patients $<45$ years, 45-60, 60-75, >75 years. Similar trend, for RSODI were found, with relatively higher value, compared to RSODS, which decreased with the increase of age. The 14-day lag estimate of CFR were 18.07 (15.6720.47), and outcome (deaths plus recoveries)-based estimate of CFR were 16.57 (14.65-18.49). The growth rate, serial interval, reproduction number and average time from onset of COVID-19 infection to death were 6.12\% (5.30\%-6.99\%), 11.4 days (9.91-12.85), 1.03 (1.01-1.05), and 11.85 days (10.55-13.15), respectively. The average daily recovery was $19.45 \%(14.75-24.15)$ and average cumulative recovery was $12.68 \%$ (10.70-14.66) among COVID-19 patients.

Conclusion: Detecting all possible cases throughout the course of the COVID-19 pandemic real CFR could be estimated to evaluate the effectiveness of healthcare systems and new treatments..

KEYWORDS: COVID-19, SARS-CoV-2, disease severity, fatality estimates, India

\section{INTRODUCTION}

In India, SARS-CoV-2, the causative agent of COVID-19, transmits with a basic reproduction number of $1.03-1.55$ $[1,2]$, and as of May 10, 2020, the country registered 2,109 people who succumbed to the disease among 62,939 confirmed cases [3]. Notably, the virus spreads very powerfully from Wuhan of Hubei province in China, the primary epicentre of COVID-19 pandemic, with basic reproduction number 2.2 [4], to the neighboring countries and beyond [5]. Alongside the reproduction number that determines the transmissibility of SARS-COV-2, CFR (case fatality rate) represents one of the most vital factors in demonstrating the severity of this novel infectious disease, critical for policy decisions on optimal healthcare facility allotment. Currently, as of May 10, 2020, based upon the total deaths as the numerator and the total confirmed cases as the denominator, also known as confirmed CFR (cCFR) of COVID-19 was 7\% globally, while the highest cCFR of $14.78 \%$ was noted for the United Kingdom, which were much higher compared to that demonstrated for India
(3.35\%-3.11\%) [6,7]. When the pandemic is still ongoing, the cCFR, is presumed to be an overvaluation of disease severity likely due to low detection of asymptomatic cases and underestimation of CFR due to the right-censoring of cases related to the time delay from symptom onset to death [8]. Also, the CFR estimates relying on the formula 'death / (recovery + death)' outpace the previous one, because of the consideration of a time delay between diagnosis and death $[9,10]$, and thus explaining that the 14-day delay estimate of CFR is not a real one in exploring COVID-19 case fatality [11, 12]. A number of studies including that we have done though demonstrated the estimation of reproduction number [1, 2], there is a dearth of reliable CFR estimates of COVID-19 ongoing pandemic particularly of Indian context. In view of the above background this communication explores various estimates of fatalities of COVID-19, along with estimation of doubling time, reproduction number, and serial interval, in estimating the COVID-19 early pandemic severity in Indian context. 


\section{METHODS}

The data on COVID-19 in India, were retrieved electronically from publicly accessible website of the Ministry of Health and Family Welfare, Government of India [3], since March 2, 2020 up to May 10, 2020. A total of 1,673,688 tests including 62,939 infected cases were recorded as of May 10, 2020. Concerning COVID-19 pandemic, India recommended testing as per the ICMR (Indian Council of Medical Research) strategy beginning March 19, 2020, that included both symptomatic and asymptomatic case contacts (laboratory confirmed and health care workers), and hospitalized severe acute respiratory illness (SARI) patients [13]. Various public and published data were used to estimate the disease severity based on information on age distribution of infected cases [14], CFR [15], and symptomatic, asymptomatic, and hospitalized cases [16].

The age-wise prevalence of COVID-19 infected cases were distributed as $67 \%, 21 \%, 10 \%$, and $2 \%$ among people $<45$ years, 45-60 years, $60-75$ years, and $>75$ years, respectively. 14 The age distribution of symptomatic CFR (sCFR), asymptomatic CFR (aCFR), hospitalized CFR (HFR) were estimated from the proportion of symptomatic (20\% of infected cases), asymptomatic $(80 \%$ of infected cases), hospitalized $(70 \%$ of symptomatic cases), and from age distribution of CFRs $(<45$ years with 15\% CFR, 45-60 years with 35\% CFR, 60-75 years with $40 \%$ CFR, and $>75$ years with $10 \%$ CFR), as publicly available and currently published data sources $[15,16]$. We estimated various COVID-19 epidemiologic parameters: growth rate, serial interval, and reproduction number, following the criteria mentioned earlier [1], and average time from onset of COVID-19 infection to death.

The estimates of cCFR (cumulative number of deaths divided by number of infected cases per day), 14-day lag estimates of CFR (cumulative number of deaths divided by number of infected cases in the past 14 days), outcome (total number of recoveries and deaths)-based CFR (cumulative number of deaths divided by number of recoveries and deaths per day). The best fitted estimates of CFR based on linear least square method were also used [17].

The sCFR was defined as the probability of dying from the infection after developing symptoms of COVID-19, while the aCFR was defined as the probability of dying from the infection but not displaying COVID-19 symptoms. The HFR was defined as the probability of dying from the infection after developing symptoms of COVID-19 and getting hospitalized. The IFR (infection fatality rate) was defined as the probability of dying from COVID-19 infection, who may or may not be symptomatic. In the present study, IFR is equivalent to CFR, because the ICMR criteria of testing included both symptomatic and asymptomatic COVID-19 cases [13]. Psym was the probability of developing symptoms after infection with COVID-19. The RSODS (relative susceptibility of developing symptoms) from asymptomatic state (assuming relative proportion of 4) was estimated as per published reports [16]. The RSODI (relative susceptibility of developing infection) from uninfected state was estimated, assuming its relative proportion of 25 among people tested for COVID-19, following ICMR criteria [18]. Overall, the cCFR, aCFR, sCFR, HFR, RSODS, and RSODI were estimated for Psym values of $0.50,0.75$, and 0.95 each for all age groups mentioned, following $\mathrm{Wu}$ et al. [19].

\section{RESULTS AND DISCUSSION}

The cCFR, aCFR, sCFR, and HFR estimates, as per our study, have been depicted in Figure 1(a) and Figure 1(b), for four different age groups and three Psym (Psym $=0.5,0.75$, $0.95)$. The overall cCFR is $2.32 \%(2.05-2.59)$, sCFR is $0.14 \%$ (0.12-0.16), HFR is $0.32 \%(0.27-0.36)$, aCFR is $1.86 \%(1.64-$ $2.07)$, values expressed as mean (95\% confidence interval). The current estimates of cCFR, aCFR, sCFR, and HFR are age as well as Psym dependent. A decrease in cCFR, aCFR, sCFR, and HFR was noticed for all Psym with the increase of age up to 75 years, while the above estimates were lowest for cases above 75 years (Figure 1). With the increase of Psym values, the cCFR, aCFR, sCFR, and HFR estimates increased gradually for all age groups ( $<45$ years to $>75$ years). However, earlier authors explained that their age-specific estimates of sCFR were Psym sensitive, while susceptibility to symptomatic infection were Psym insensitive, in relation to the study of COVID-19 pandemic in China [19]. Age distribution of cCFR of COVID-19 in India showed that though highest cCFR (40\%) was seen among people of 60-75 years age group, the CFR, aCFR, sCFR, and HFR were 50 times higher among people $<60$ years compared to the older population above 60 years. Herein, the highest prevalence of COVID-19 infection was estimated to be $67 \%$ in India among people $<45$ years, which was $\sim 34$ times higher than those $<75$ years, following published information of cases [14].

The relative susceptibility estimates of developing symptoms and infection due to COVID-19 are represented in Figure 2(a) and Figure 2(b) respectively. The relative susceptibility estimates of developing symptoms due to COVID-19 disease were 1.97 (0.47-3.47), 0.62 (0.15-1.09), $0.29(0.07-0.52), 0.06(0.02-0.10)$ respectively for $<45$ years, $45-60,60-75,>75$ years of age groups. Similar trend, for the relative susceptibility estimates of developing COVID-19 infection, were found for all Psym and age groups, with relatively higher value, compared to the relative susceptibility of developing symptoms, which decreased with the increase of age as well as Psym (Figure 2). Our estimates revealed that the RSODI was $\sim 6$ times higher than RSODI among all cases, the RSODS and RSODI being four and 25 respectively from asymptomatic and uninfected condition respectively, that is, prevalence of four asymptomatic people over one symptomatic person, and one infected case prevails among 25 uninfected people, respectively. In our estimate the RSODS and RSODI both increased from the baseline Psym $=0.5$ to Psym $=0.95$ at the top level. The RSODS values were 0.16 


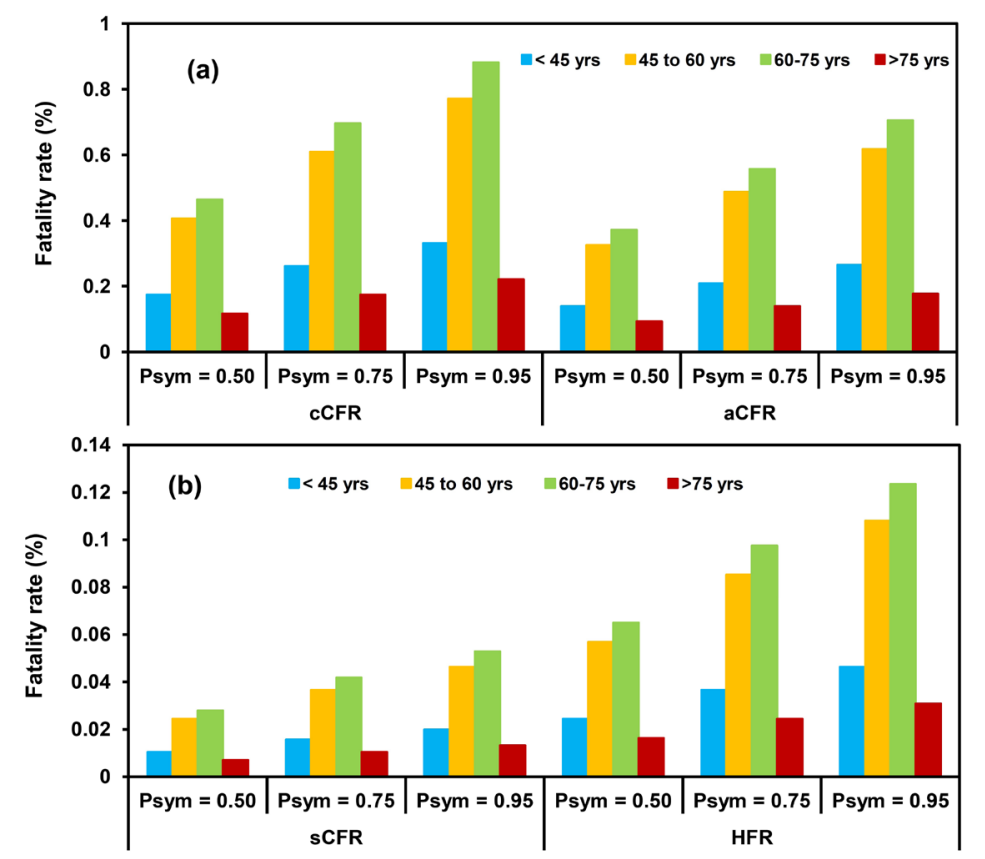

Figure 1: COVID-19 fatality rate estimates for four different age groups and three Psym (Psym: 0.5, 0.75, 0.95): (a) confirmed CFR (cCFR) and asymptomatic CFR (aCFR); (b) symptomatic CFR (sCFR) and hospitalized CFR (HFR). Psym: probability of developing symptoms.
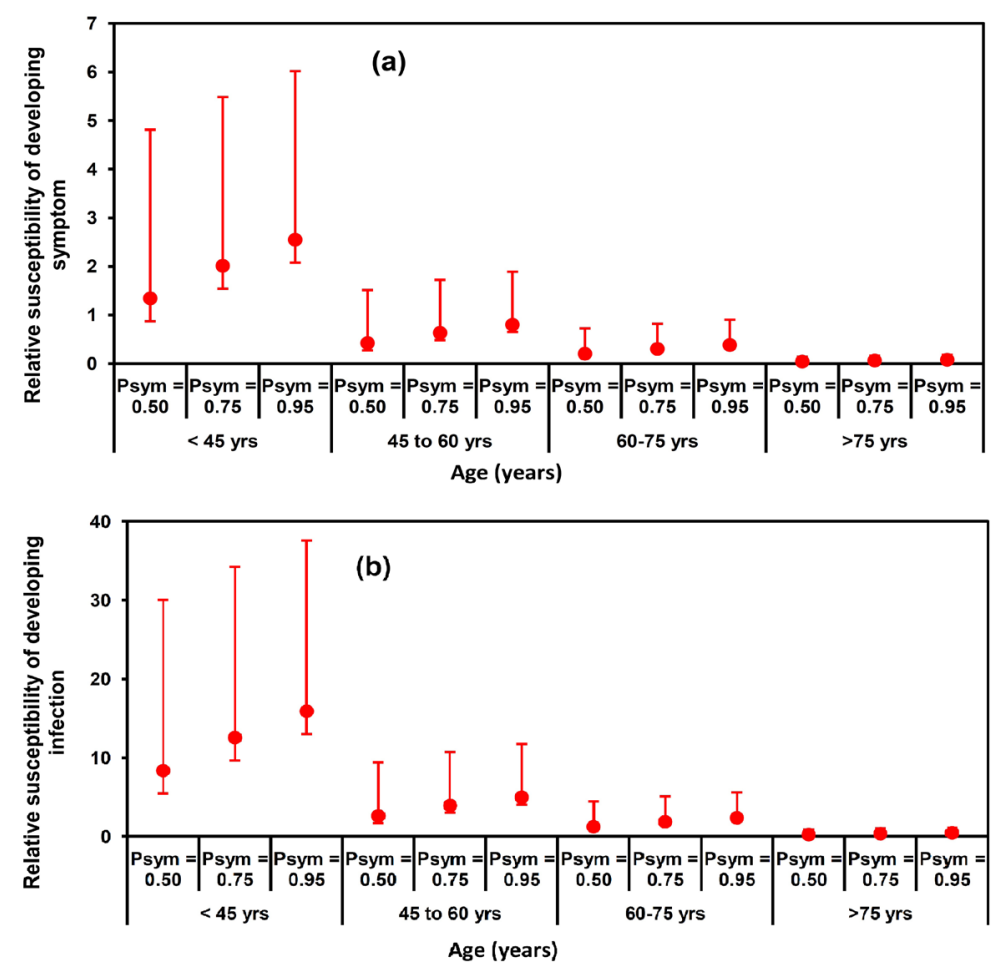

Figure 2: COVID-19 relative susceptibility estimates: (a) developing symptoms; (b) developing infection. Psym: probability of developing symptoms. 
and two times higher among patients of $<30$ years and $>59$ years, respectively, compared to those aged 30-59 years, as has been explained by $\mathrm{Wu}$ et al. [19].

The CFR estimates in the Diamond Princess ship and in China were $2.6 \%$ and $1.2 \%$ as of February 2020 [20]. Figure 3 demonstrates different estimates of COVID-19 fatalities in India. The values of 14-day lag estimate of CFR were 18.07 (15.67-20.47) and outcome (deaths plus recoveries) based estimate of CFR were 16.57 (14.65-18.49). The outcomebased and 14-day lag estimates of CFR were, respectively, $\sim 7$ and $\sim 8$ times higher than cCFR estimate [2.32 (2.05-2.59)]. In China, the age adjusted CFR (ACFR) was $3.8 \%$ as of February 22, 2020, and in Italy, the ACFR ranged 4.7\%-7\% as of March 20,2020 [21], both of which were lower than the ACFR value of $15 \%(1.58-48.42)$ in India, as estimated in the present study. The outcome-based CFR estimate was applied for SARS by Ghani et al. [22], and that was independent of any window period providing point-estimate during the course of epidemic.

The best linear fitted CFR estimates by confirmed cases were $3.32 \%(\mathrm{R} 2=0.9988)$, 14-day lag estimates were $7.8 \%$ $(\mathrm{R} 2=0.9784)$ and by outcome based estimates were $10.27 \%$ $(\mathrm{R} 2=0.9806)$, as represented in the insets of Figure 3. Considering the 14-day delay from confirmation to death, the time-dependent average CFR estimate was two times less than the overall 14-day delay CFR estimate by regression analysis. Similar method was used by Yang et al. [17], and Oztoprak et al. [23], in estimating CFR for COVID-19 in different geographical regions. Considering the population density of 19 Indian states and union territories displaying deaths as of May 1, 2020, the population-adjusted CFR in India was $0.17 \%$, in our study, which provide an estimate of the COVID-19 CFR in India at the population level; such CFR was estimated by employing population adjusted number of COVID-19 cases, as reported by Iype and Gulati [24].

The difference in CFR estimates were plausibly due to the presence of comorbidities (diabetes mellitus, chronic obstructive pulmonary disease, coronary artery disease, hypertension), which in Indian situation represent $78 \%$ of the COVID-19 infection [15]; furthermore, the hospitalized cases $(70 \%$ of symptomatic infection), of which $15 \%$ leading into severe ICU admission, could presumably overvalue the $\mathrm{CFR}$, if mild or asymptomatic cases were not detected. The incomplete testing as well as reporting of deaths, if any, were accounted by estimating CFR with a time delay from infection onset to death, which in our estimate was 11.85 days (Figure 5). Several other factors can result in CFR disparities owing to uncertainty related to case definition, right-censoring of cases, and the untraceable cases [24].

The CFR estimates can be biased upwards by underreporting of cases and downwards by failure in accounting the delay from case confirmation to death. However, this might not be true for COVID-19 pandemic situation in India so far the ICMR testing strategy comprises both symptomatic and asymptomatic cases infected with COVID-19 [13]. Moreover, the serial interval estimates ( 3.5 days) indicate the presence of only asymptomatic COVID-19 infection for two days (March 2, 2020, and March 3, 2020). Besides, based upon the SI values of 7.8 days, for other days prior to the commencing date of ICMR testing that was on March 19, 2020, the infected cases in India were all symptomatic in nature [25].

Figure 4 displays the Psym-variable current estimates of the major epidemiologic factors of COVID-19 pandemic in Indian context. The growth rate, serial interval, and reproduction

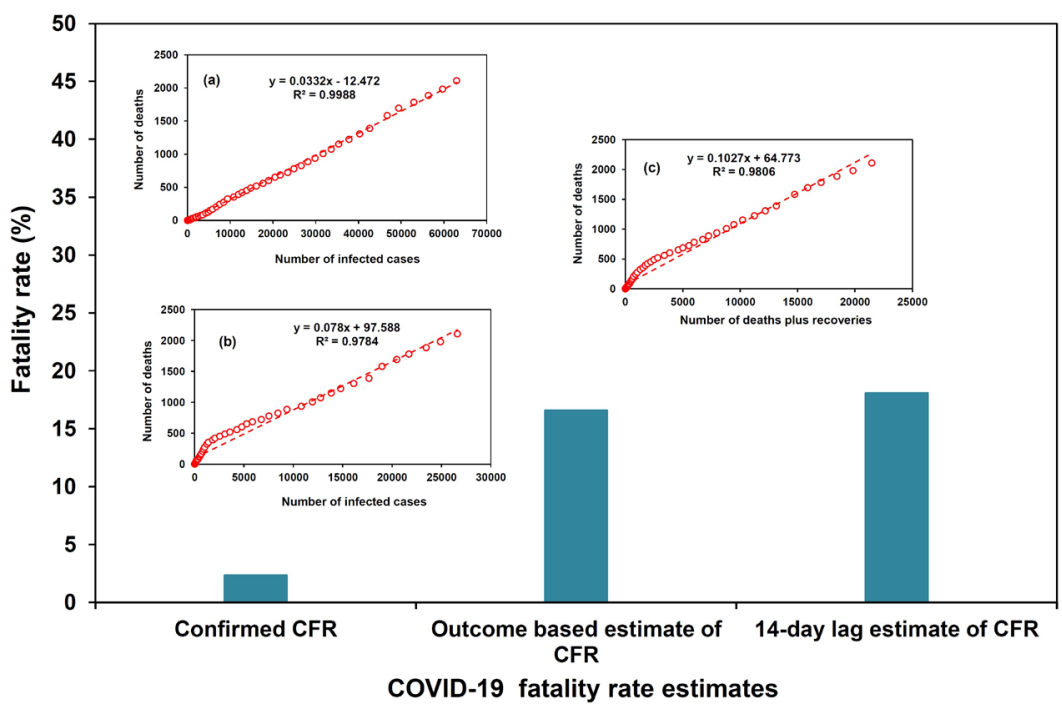

Figure 3: COVID-19 CFR and linear fitted estimates based on: (a) confirmed cases, (b) 14-day lag, (c) outcome (deaths plus recoveries). 
number and average time from onset of COVID-19 infection to death, were $6.12 \%(5.30 \%-6.99 \%), 11.4$ days (9.91-12.85), 1.03 (1.01-1.05), and 11.85 days (10.55-13.15), respectively. As per the ICMR status update on SARS-CoV-2 (COVID-19) testing [18], a total of 1,673,688 samples were tested out of which $3.76 \%$ (62939) tested positive for COVID-19 (Figure 5) accounting for 1228 tests per one million population of
India, as of May 10, 2020. As per our estimates, the average 4.84\% (4.27- 5.41) COVID-19 cumulative infected cases and $3.69 \%(3.33-4.06)$ daily infected cases against the tests were recorded, while following regression analysis the values were respectively, $3.68 \%(\mathrm{R} 2=0.9975)$ and $3.12 \%(\mathrm{R} 2=0.8697)$ (Figure 5).

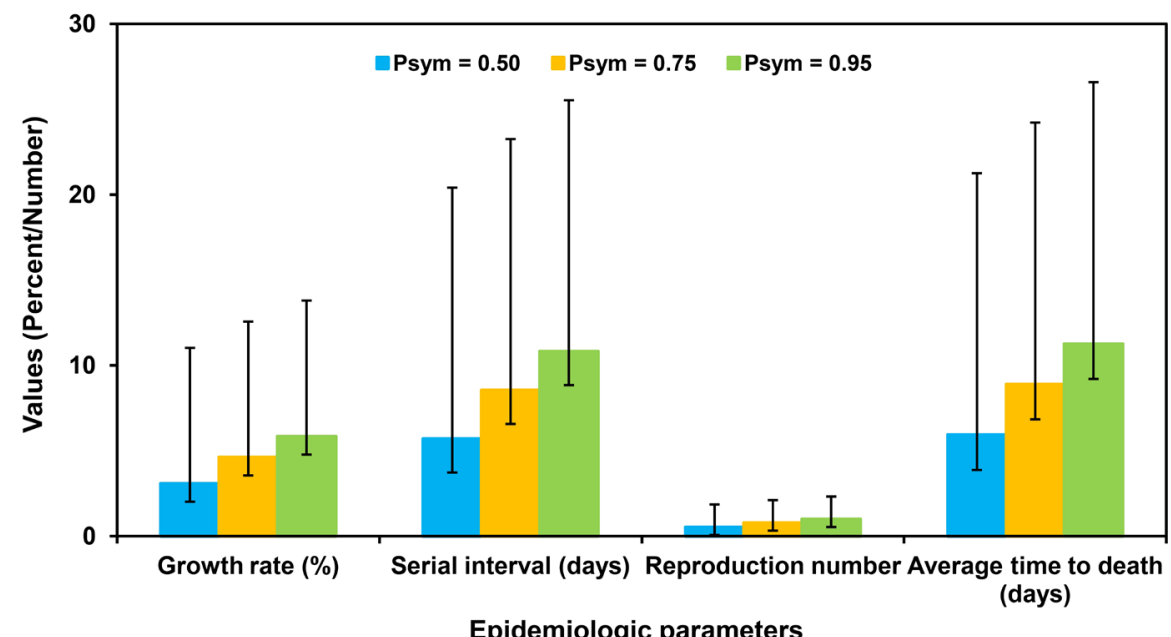

Figure 4: $P_{\text {sym }}$ (probability of developing symptoms)-variable estimates of epidemiologic factors of COVID-19 pandemic in India: growth rate, serial interval, reproduction number and average time from onset of COVID-19 infection to death.

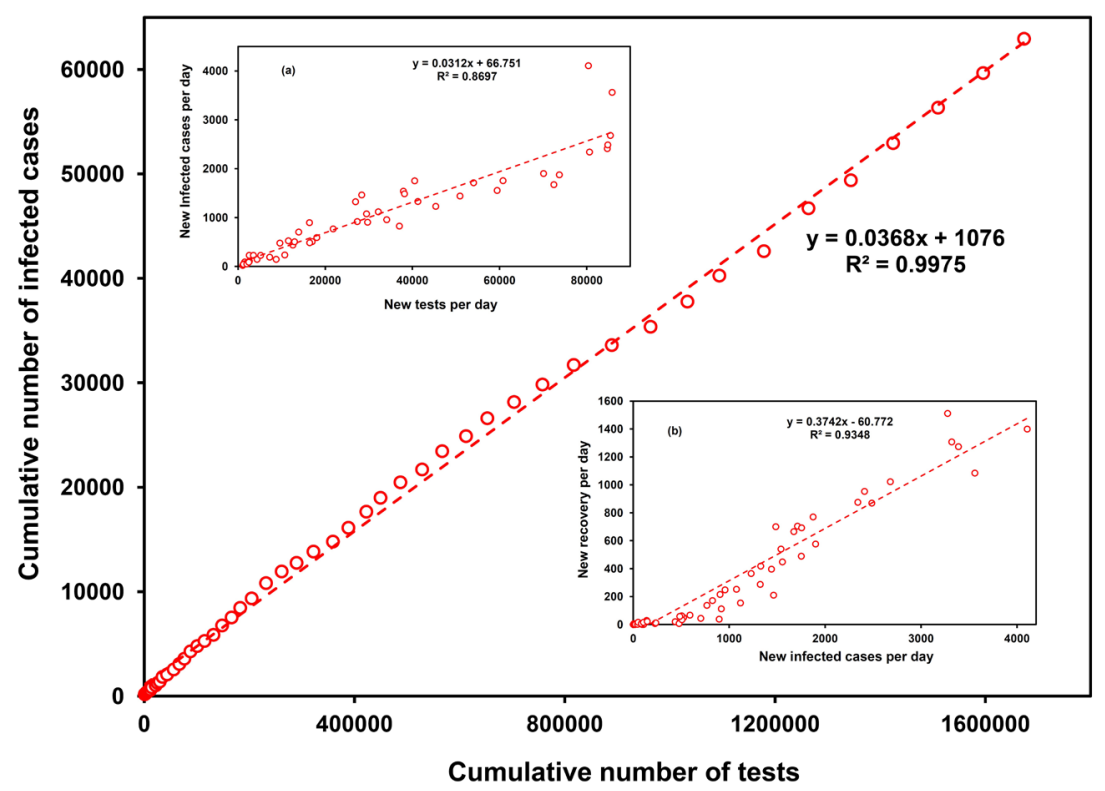

Figure 5: Cumulative tests versus COVID-19 infected cases in India. Insets display (a) new tests versus new infected cases per day, and (b) new infected cases versus new recoveries per day. 
According to the published data on the facility preparedness of the states/union territories governments as well as the central government on COVID-19 in India, there have been 7,740 facilities in 483 districts to support COVID-19, a total of 305,567 beds for confirmed cases, 99,492 oxygen supported beds, 34,076 ICU beds, in all the states/union territories, as of May 10, 2020 [26, 27], for an infection attack rate of 465 per million as per the current estimation, following the criteria of Centers for Disease Control and Prevention [28]. Such medical resources and healthcare setup achieved average daily recovery of $19.45 \%$ (14.75- 24.15) (Figure 5) and average cumulative recovery of $12.68 \%$ (10.70- 14.66) COVID-19 patients, with overall best-fitted estimates of $37.42 \%$ (R2 = $0.9348)$ and $28.34 \%(\mathrm{R} 2=0.9706)$ respectively, as we have estimated. As per our belief and knowledge, this is the first study of its kind in reporting COVID-19 severity in Indian context during early pandemic.

\section{CONCLUSION}

Accounting the symptomatic as well as asymptomatic cases in testing COVID-19 infection, the dynamic CFR estimation upon confirmed cases reflect the severity of this emerging infectious disease overtime. With a rise in effective testing through contact tracing and survey of infection prevalence as well as detecting all possible cases throughout the course of the pandemic real CFR could be estimated to evaluate the effectiveness of healthcare systems and new treatments.

\section{ACKNOWLEDGEMENT}

The corresponding author acknowledges Research Square for posting preprint of the article with DOI: 10.21203/ rs.3.rs-29484/v1

\section{Conflicts of Interest}

We declare that there is no conflict of interest.

\section{Funding support}

There was no source of funding for this study.

\section{REFERENCES}

1. Mandal M, Mandal S. COVID-19 pandemic scenario in India compared to China and rest of the world: a data driven and model analysis. medRxiv 2020. DOI: $10.1101 / 2020.04 .20 .20072744$

2. Tiwari A. Modelling and analysis of COVID-19 epidemic in India. medRxiv 2020. DOI: 10.1101/2020.04.12.20062794

3. Ministry of Health and Family Welfare, Government of India. COVID-19 India. Available from: https://www.mohfw.gov.in/index.php (Accessed on May 10, 2020).

4. Sanche S, Lin YT, Xu C, Romero-Severson E, Hengartner N, Ke R. High contagiousness and rapid spread of severe acute respiratory syndrome coronavirus 2. Emerging Infectious Diseases. 2020; 26(7):1470-7.

5. World Health Organization. Coronavirus disease (COVID-2019) situation reports. WHO 2020. Available from: https://www.who.int/emergencies/ diseases/novel-coronavirus-2019/situation-reports. [Accessed on May 10, 2020]

6. Our World in Data. Case fatality rate of the ongoing COVID-19 pandemic. Available from: https://ourworldindata.org/grapher/coronavirus-cfr
(Accessed on May 11, 2020).

7. Mandal M, Mandal S. Phasic containment of COVID-19 in substantially affected states of India. medRxiv 2020.DOI: 10.1101/2020.05.05.20092130

8. Rajgor DD, Lee MH, Archuleta S, Bagdasarian N, Quek SC. The many estimates of the COVID-19 case fatality rate. Lancet Infectious Diseases. 2020; 20(7): 776-7.

9. Wilson N, Kvalsvig A, Barnard LT, Baker GM. Case-fatality estimates for COVID-19 calculated by using a lag time for fatality. Emerging Infectious Diseases. 2020; 26(6):1339-1441.

10. Baud D, Qi X, Nielsen-Saines K, Musso D, Pomar L, Favre G, et al. Real estimates of mortality following COVID-19 infection. Lancet Infectious Diseases. 2020; 20(7):773.

11. Spychalski P, Blazynska-Spychalska A, Kobiela J. Estimating case fatality rates of COVID-19. Lancet Infectious Diseases. 2020; 20(7):774-775.

12. Lipsitch M. Estimating case fatality rates of COVID-19. Lancet Infectious Diseases. 2020; 20(7):775.

13. WHO India. Novel coronavirus disease (COVID-19) situation update report-8. Published online March 22, 2020. Available from: https://www. who.int/india/emergencies/india-situation-report (Accessed on March 23, 2020).

14. Statista, Germany. COVID-19. Available from: https://www.statista. com/statistics/1110522/India-number-of-coronavirus-cases-by-age-group (Accessed May 14, 2020).

15. Indian Express, India. Covid-19: Almost 50\% of the dead are below age 60 , up from $25 \%$. Available from: https://www.indianexpress.com (Accessed on May 1, 2020).

16. India Today, India. Coronavirus: $80 \%$ cases asymptomatic, but no need to revise testing criteria, says ICMR. Available from: https://www. indiatoday.in (Accessed on April 20, 2020).

17. Yang S, Cao P, Du P, Wu Z, Zhuang Z, Yang L, et al. Early estimation of the case fatality rate of COVID-19 in mainland China: a data-driven analysis. Annals of Translational Medicine. 2020; 8: 128.

18. Indian Council of Medical Research. SARS-CoV-2 (COVID-19) testing status. ICMR 2020. (Published online May 10, 2020). Available from: https://www.icmr.gov.in (Accessed on May 10, 2020).

19. Wu JT, Leung K, Bushman M, Kishore N, Niehus R, de Salazar PM, et al. Estimating clinical severity of COVID-19 from the transmission dynamics in Wuhan, China. Nature Medicin.e 2020; 26: 506-10.

20. Russell TW, Hellewell J, Jarvis CI, van Zandvoort K, Abbott S, Ratnayake $\mathrm{R}$ et al. Estimating the infection and case fatality ratio for coronavirus disease (COVID-19) using age-adjusted data from the outbreak on the Diamond Princess cruise ship. Eurosurveillance. 2020; 25: 1-5.

21. Bignami S, Ghio D. A demographic adjustment to improve measurement of COVID-19 severity at the developing stage of the pandemic. medRxiv 2020. DOI: $10.1101 / 2020.03 .23 .20040998$

22. Ghani AC, Donnelly CA, Cox DR, Griffin JT, Fraser C, Lam TH, et al. Methods for estimating the case fatality ratio for a novel. Emerging Infectious Disease. American Journal of Epidemiology. 2005; 162: 47986.

23. Oztoprak F, Javed A. Case fatality rate estimation of COVID-19 for European countries: Turkey's current scenario amidst a global pandemic; comparison of outbreaks with European countries. EJMO. 2020; 4: 14959.

24. Iype E, Gulati S. Understanding the asymmetric spread and case fatality rate (CFR) for COVID-19 among countries. medRxiv 2020. DOI: 10.1101/2020.04.21.20073791

25. WHO India. Novel coronavirus disease (COVID-19) situation update report-12. Published online April 19, 2020. Available from: https://www. who.int/india/emergencies/india-situation-report (Accessed on April 20, 2020).

26. WHO India. Novel coronavirus disease (COVID-19) situation update report-15. Published online May 10, 2020. Available from: https://www. who.int/india/emergencies/india-situation-report (Accessed on May 11, 2020). 
27. Ministry of Health and Family Welfare, Government of India. Guidance document on appropriate management of suspect/ confirmed cases of COVID-19. MHFW-GoI 2020. (Published online April 7, 2020). Available from: https://www.mohfw.gov.in/pdf/ FinalGuidanceonMangaementofCovidcasesversion2.pdf (Accessed on April 20, 2020).
28. Centers for Disease Control and Prevention. Principles of Epidemiology in Public Health Practice. CDC 2012. Available from: https://www.cdc. gov/csels/dsepd/ss1978/SS1978.pdf (Accessed on May 15, 2020).

Citation: Manisha Mandal, Shyamapada Mandal, “Estimating COVID-19 Early Pandemic Severity in Indian Context”, American Research Journal of Biosciences, vol 6, no. 1, 2020, pp. 1-7.

Copyright (c) 2020 Prof. Shyamapada Mandal, et al. This is an open access article distributed under the Creative Commons Attribution License, which permits unrestricted use, distribution, and reproduction in any medium, provided the original work is properly cited. 